\title{
Growth of Sobolev norms and controllability of Schrödinger equation
}

\author{
Vahagn Nersesyan \\ Laboratoire de Mathématiques, Université de Paris-Sud XI \\ Bâtiment 425, 91405 Orsay Cedex, France \\ E-mail: Vahagn.Nersesyan@math.u-psud.fr
}

\begin{abstract}
In this paper we obtain a stabilization result for the Schrödinger equation under generic assumptions on the potential. Then we consider the Schrödinger equation with a potential which has a random time-dependent amplitude. We show that if the distribution of the amplitude is sufficiently non-degenerate, then any trajectory of system is almost surely non-bounded in Sobolev spaces.
\end{abstract}

\section{Contents}

1 Introduction 2

2 Preliminaries 4

3 Controllability of the Schrödinger equation 4

3.1 Stabilization result . . . . . . . . . . . . . . 4

3.2 Approximate controllability . . . . . . . . . . . . . 7

3.3 Proof of Theorem $3.3 . \ldots \ldots \ldots$

3.4 Genericity of Condition $3.2 \ldots \ldots \ldots \ldots$

4 Applications 13

4.1 Nonlinear Schrödinger equation _. . . . . . . . . . 13

4.2 Randomly forced Schrödinger equation . . . . . . . . 15

4.2.1 Growth of Sobolev norms . . . . . . . . . . . . 15

4.2 .2 Proof of Theorem $4.7 \ldots \ldots \ldots \ldots$

$\begin{array}{ll}\text { References } & \mathbf{1 7}\end{array}$ 


\section{Introduction}

We consider the problem

$$
\begin{aligned}
i \dot{z} & =-\Delta z+V(x) z+u(t) Q(x) z, \quad x \in D, \\
\left.z\right|_{\partial D} & =0, \\
z(0, x) & =z_{0}(x),
\end{aligned}
$$

where $D \subset \mathbb{R}^{m}$ is a bounded domain with smooth boundary, $V, Q \in C^{\infty}(\bar{D}, \mathbb{R})$ are given functions, $u$ is the control, and $z$ is the state. Under some hypotheses on $V$ and $Q$ (see Condition [3.2), we prove a stabilization result for problem (1.1), (1.2). Then this result is applied to show that almost any trajectory of random Schrödinger equation is non-bounded in Sobolev spaces. As is shown in Section 3.4, the hypotheses on $V$ and $Q$ are in a sense generic.

Let us recall some previous results on controllability of Schrödinger equation. A general negative result for bilinear control systems is obtained by Ball, Marsden and Slemrod [5. Application of this result to (1.1), (1.2) implies that the set of attainable points from any initial data in $H^{2}$ admits a dense complement in $H^{2}$. We refer the reader to the papers [25, 27, 4, 3, 1] and the references therein for controllability of finite-dimensional systems. In [7], Beauchard proves that exact controllability result is possible to obtain if one chooses properly the phase space. More precisely, in the case $m=1, V(x)=0$ and $Q(x)=x$ exact controllability of the problem is proved in $H^{7}$-neighborhood of the eigenstates. A stabilization property for finite-dimensional approximations of Schrödinger equation is obtained by Beauchard et al., in [8], which was later generalized by Beauchard and Mirrahimi 9 to the infinite-dimensional case for $m=1, V(x)=0$ and $Q(x)=x$ (see also the paper by Mirrahimi [21]). Recently Chambrion et al. [13, under some assumptions on $V, Q \in C^{\infty}(\bar{D}, \mathbb{R})$, derived the approximate controllability of (1.1), (1.2) in $L^{2}$ from the controllability of finite-dimensional projections. See also the papers [19, 11, 20, 6, 28, 15] and the references therein for controllability results by boundary controls and controls supported in a given subdomain and the book [14] by Coron for introduction to the later developments and methods in the control theory of nonlinear systems.

The main result of this paper states that any neighborhood of the first eigenfunction of operator $-\Delta+V$ is attainable from any initial point $z_{0} \in H^{2}$. This result, combined with the time reversibility property of the system and the fact that the equation is linear, implies approximate controllability property in $L^{2}$.

Let us describe in a few words the main ideas of the proof. As $V, Q$ and $u$ are real-valued, the $L^{2}$ norm is preserved by the flow of the system. Thus it suffices to consider the restriction of (1.1), (1.2) to the unit sphere $S$ in $L^{2}$. We introduce a Lyapunov function $\mathcal{V}(z)$ that controls the $H^{2}$-norm of $z$. The infimum of $\mathcal{V}$ on the sphere $S$ is attained at the first eigenfunction $e_{1, V}$ of the operator $-\Delta+V$. Using the idea of generating trajectories with Lyapunov techniques from [8], we choose a feedback law $u(z)$ such that the function $\mathcal{V}$ 
decreases on the solutions of the corresponding system:

$$
\mathcal{V}\left(\mathcal{U}_{t}\left(z_{0}, u\right)\right)<\mathcal{V}\left(z_{0}\right), \quad t>0,
$$

where $\mathcal{U}_{t}(\cdot, u)$ is the resolving operator of (1.1), (1.2). Then iterating this construction and using the fact that the system is autonomous, we prove that the $H^{2}$-weak $\omega$-limit set of any solution contains the minimum point of function $\mathcal{V}$, i.e. the eigenfunction $e_{1, V}$ (see Sections 3.1 and 3.3).

The ideas of the proof work also in the case of nonlinear equation. We think that the result holds also in the spaces $H^{l}, l>2$. This case will be treated in a later paper.

We next use the above-mentioned controllability result to study the large time behavior of solutions of random Schrödinger equation. We show that if the distribution of the random potential is sufficiently non-degenerate (see Condition 4.6), then the trajectories of the system are almost surely non-bounded. It is interesting to compare this result with that of Eliasson and Kuksin [16, where KAM-technique is applied to prove the reducibility of a linear Schrödinger equation with time-quasiperiodic potential. In particular, it is proved that for most values of the frequency vector the Sobolev norms of the solutions are bounded. Examples of non-bounded solutions of 1D linear Schrödinger equation with some random potentials are constructed in [10, 17, where also the growth rate estimates are given. Our assumptions on the distribution of the potential are more general, and the proof works also in the case of nonlinear equation. However, at this level of generality, we do not have any lower bound on the rate of growth of Sobolev norms.

The idea of the proof is to show that the first entrance time to any ball centered at the origin in $H^{-\varepsilon}$ is almost surely finite. This implies immediately that almost any trajectory of the system approaches the origin arbitrarily closely in $H^{-\varepsilon}$. Combining this with the fact that the $L^{2}$-norm is preserved, we conclude that almost any trajectory is non-bounded in $H^{l}$ for any $l>0$.

In conclusion, let us note that the results of this paper imply the irreducibility in $L^{2}$ of the Markov chain associated with (1.1). This property is not sufficient to prove the ergodicity of the dynamics generated by the Schrödinger equation with random potential. However, in the case of finite-dimensional approximations, that question is treated in the paper [22, in which an exponential mixing property is established. We hope the methods developed in this work will help to tackle the infinite-dimensional case.

Acknowledgments. The author would like to thank Armen Shirikyan for his guidance and encouragements.

\section{Notation}

In this paper we use the following notation. Let $D \subset \mathbb{R}^{m}, m \geq 1$ be a bounded domain with smooth boundary. Let $H^{s}:=H^{s}(D)$ be the Sobolev space of order 
$s \in \mathbb{R}$ endowed with the norm $\|\cdot\|_{s}$. Consider the operators $-\Delta z+V z, z \in$ $\mathcal{D}(-\Delta+V):=H_{0}^{1} \cap H^{2}$, where $V \in C^{\infty}(\bar{D}, \mathbb{R})$. We denote by $\left\{\lambda_{j, V}\right\}$ and $\left\{e_{j, V}\right\}$ the sets of eigenvalues and normalized eigenfunctions of $-\Delta+V$. Let $\langle\cdot, \cdot\rangle$ and $\|\cdot\|$ be the scalar product and the norm in the space $L^{2}$. Let $S$ be the unit sphere in $L^{2}$. For a Banach space $X$, we shall denote by $B_{X}(a, r)$ the open ball of radius $r>0$ centered at $a \in X$. The set of irrational numbers is denoted by $\mathbb{I}$.

\section{Preliminaries}

The following lemma shows the well-posedness of system (1.1)-(1.3).

Lemma 2.1. For any $z_{0} \in H_{0}^{1} \cap H^{2}\left(z_{0} \in L^{2}\right)$ and for any $u \in L_{l o c}^{1}([0, \infty), \mathbb{R})$ problem (1.1)-(1.3) has a unique solution $z \in C\left([0, \infty), H^{2}\right)\left(z \in C\left([0, \infty), L^{2}\right)\right)$. Furthermore, the resolving operator $\mathcal{U}_{t}(\cdot, u): L^{2} \rightarrow L^{2}$ taking $z_{0}$ to $z(t)$ satisfies the relation

$$
\left\|\mathcal{U}_{t}\left(z_{0}, u\right)\right\|=\left\|z_{0}\right\|, \quad t \geq 0 .
$$

See 12 for the proof. Notice that the conservation of $L^{2}$-norm implies that it suffices to consider the controllability properties of (1.1), (1.2) on the unit sphere $S$.

In Section 4.2 we replace the control $u$ by a random process. Namely, we consider the equation

$$
i \dot{z}=-\Delta z+V(x) z+\beta(t) Q(x) z, \quad x \in D,
$$

where $\beta(t)$ is a random process of the form

$$
\beta(t)=\sum_{k=0}^{+\infty} I_{k}(t) \eta_{k}(t-k), \quad t \geq 0 .
$$

Here $I_{k}(\cdot)$ is the indicator function of the interval $[k, k+1)$ and $\eta_{k}$ are independent identically distributed (i.i.d.) random variables in $L^{2}([0,1], \mathbb{R})$.

Let $z_{0}$ be $L^{2}$-valued random variable independent of $\left\{\eta_{k}\right\}$. Denote by $\mathcal{F}_{k}$ the $\sigma$-algebra generated by $z_{0}, \eta_{0}, \ldots, \eta_{k-1}$.

Lemma 2.2. Under above conditions, $\mathcal{U}_{k}(\cdot, \beta)$ is a homogeneous Markov chain with respect to $\mathcal{F}_{k}$.

This lemma is proved by standard arguments (e.g., see [23]).

\section{Controllability of the Schrödinger equation}

\subsection{Stabilization result}

Let us introduce the Lyapunov function

$$
\mathcal{V}(z):=\alpha\left\|(-\Delta+V) P_{1, V} z\right\|^{2}+1-\left|\left\langle z, e_{1, V}\right\rangle\right|^{2}, \quad z \in S \cap H_{0}^{1} \cap H^{2},
$$


where $\alpha>0$ and $P_{1, V} z:=z-\left\langle z, e_{1, V}\right\rangle e_{1, V}$ is the orthogonal projection in $L^{2}$ onto the closure of the vector span of $\left\{e_{k, V}\right\}_{k \geq 2}$. Notice that $\mathcal{V}(z) \geq 0$ for all $z \in S \cap H_{0}^{1} \cap H^{2}$ and $\mathcal{V}(z)=0$ if and only if $z=c e_{1, V},|c|=1$. For any $z \in S \cap H_{0}^{1} \cap H^{2}$, we have

$$
\mathcal{V}(z) \geq \alpha\left\|(-\Delta+V) P_{1, V} z\right\|^{2} \geq \frac{\alpha}{2}\left\|\Delta\left(P_{1, V} z\right)\right\|^{2}-C_{1} \geq \frac{\alpha}{4}\|\Delta z\|^{2}-C_{2} .
$$

Thus

$$
C(1+\mathcal{V}(z)) \geq\|z\|_{2}
$$

for some constant $C>0$. Following the ideas of [8], we wish to choose a feedback law $u(\cdot)$ such that

$$
\frac{\mathrm{d}}{\mathrm{d} t} \mathcal{V}(z(t)) \leq 0
$$

for the solution $z(t)$ of (1.1)-(1.3). Let us assume that $\Delta z(t) \in H_{0}^{1} \cap H^{2}$ for all $t \geq 0$. Using (1.1), we get

$$
\begin{aligned}
\frac{\mathrm{d}}{\mathrm{d} t} \mathcal{V}(z(t))= & 2 \alpha \operatorname{Re}\left(\left\langle(-\Delta+V) P_{1, V} \dot{z},(-\Delta+V) P_{1, V} z\right\rangle\right)-2 \operatorname{Re}\left(\left\langle\dot{z}, e_{1, V}\right\rangle\left\langle e_{1, V}, z\right\rangle\right) \\
= & 2 \alpha \operatorname{Re}\left(\left\langle(-\Delta+V) P_{1, V}(i \Delta z-i V z-i u Q z),(-\Delta+V) P_{1, V} z\right\rangle\right) \\
& -2 \operatorname{Re}\left(\left\langle i \Delta z-i V z-i u Q z, e_{1, V}\right\rangle\left\langle e_{1, V}, z\right\rangle\right) .
\end{aligned}
$$

Integrating by parts and using the fact that

$$
\left.(-\Delta+V) P_{1, V} z\right|_{\partial D}=\left.z\right|_{\partial D}=\left.e_{1, V}\right|_{\partial D}=0,
$$

we obtain

$$
\begin{aligned}
2 \alpha & \operatorname{Re}\left(\left\langle-i(-\Delta+V)^{2} P_{1, V} z,(-\Delta+V) P_{1, V} z\right\rangle\right)-2 \operatorname{Re}\left(\left\langle i \Delta z-i V z, e_{1, V}\right\rangle\left\langle e_{1, V}, z\right\rangle\right) \\
= & 2 \alpha \operatorname{Re}\left(\left\langle i \nabla(-\Delta+V) P_{1, V} z, \nabla(-\Delta+V) P_{1, V} z\right\rangle\right) \\
& +2 \alpha \operatorname{Re}\left(\left\langle-i V(-\Delta+V) P_{1, V} z,(-\Delta+V) P_{1, V} z\right\rangle\right) \\
& +2 \lambda_{1, V} \operatorname{Re}\left(\left\langle i z, e_{1, V}\right\rangle\left\langle e_{1, V}, z\right\rangle\right)=0 .
\end{aligned}
$$

Thus

$\frac{\mathrm{d}}{\mathrm{d} t} \mathcal{V}(z(t))=2 u \operatorname{Im}\left(\alpha\left\langle(-\Delta+V) P_{1, V}(Q z),(-\Delta+V) P_{1, V} z\right\rangle-\left\langle Q z, e_{1, V}\right\rangle\left\langle e_{1, V}, z\right\rangle\right)$.

Let us take

$$
u(z):=-\delta \operatorname{Im}\left(\left\langle\alpha(-\Delta+V) P_{1, V}(Q z),(-\Delta+V) P_{1, V} z\right\rangle-\left\langle Q z, e_{1, V}\right\rangle\left\langle e_{1, V}, z\right\rangle\right),
$$

where $\delta>0$ is a small constant. Then

$$
\frac{\mathrm{d}}{\mathrm{d} t} \mathcal{V}(z(t))=-\frac{2}{\delta} u^{2}(z(t))
$$

Consider the equation

$$
i \dot{z}=-\Delta z+V(x) z+u(z) Q(x) z, \quad x \in D .
$$


Proposition 3.1. For any $z_{0} \in H_{0}^{1} \cap H^{2}$ problem (3.4), (1.2), (1.3) has a unique solution $z \in C\left([0, \infty), H_{0}^{1} \cap H^{2}\right)$. Moreover, the following properties hold.

(i) If $\Delta z_{0} \in H_{0}^{1} \cap H^{2}$, then $\Delta z \in C\left([0, \infty), H_{0}^{1} \cap H^{2}\right)$.

(ii) Let $\mathcal{U}_{t}(\cdot): H_{0}^{1} \cap H^{2} \rightarrow H_{0}^{1} \cap H^{2}$ be the resolving operator. If $T>0$, $z_{n} \in H_{0}^{1} \cap H^{2}$ and $z_{n} \rightarrow z_{0}$ in $H^{2}$, then $\mathcal{U}_{T}\left(z_{n_{k}}\right) \rightarrow \mathcal{U}_{T}\left(z_{0}\right)$ in $H^{2}$ for some sequence $k_{n} \geq 1$.

Sketch of the proof. The local well-posedness of (3.4), (1.2) and (1.3) is standard (see [12]). From the construction of the feedback $u$ it follows that a finite-time blow-up is impossible. Hence the solution is global in time. To prove the rest of the theorem, it suffices to show that $u\left(z_{n}\right) \rightarrow u\left(z_{0}\right)$ for any $z_{n} \in H_{0}^{1} \cap H^{2}$ such that $z_{n} \rightarrow z_{0}$ in $H^{2}$. Notice that (3.2) and the fact that $Q$ is real imply that

$$
u(z)=-\operatorname{Im}(\langle\alpha Q(-\Delta+V) z,(-\Delta+V) z\rangle+\tilde{u}(z)=\tilde{u}(z),
$$

where $\tilde{u}\left(z_{n}\right) \rightarrow \tilde{u}\left(z_{0}\right)$. This completes the proof.

Thus if $z_{0}, \Delta z_{0} \in H_{0}^{1} \cap H^{2}$, then (3.3) is verified for $z(t)=\mathcal{U}_{t}\left(z_{0}\right)$. A density argument proves the identity for any $z_{0} \in H_{0}^{1} \cap H^{2}$.

Let us assume that the functions $V$ and $Q$ satisfy the following condition.

Condition 3.2. The functions $V, Q \in C^{\infty}(\bar{D}, \mathbb{R})$ are such that:

(i) $\left\langle Q e_{1, V}, e_{j, V}\right\rangle \neq 0$ for all $j \geq 1$,

(ii) $\lambda_{1, V}-\lambda_{j, V} \neq \lambda_{p, V}-\lambda_{q, V}$ for all $j, p, q \geq 1$ such that $\{1, j\} \neq\{p, q\}$ and $j \neq 1$.

The below theorem is the main result of this section.

Theorem 3.3. Under Condition 3.2, there is a finite or countable set $J \subset \mathbb{R}_{+}^{*}$ such that for any $\alpha \notin J$ and $z_{0} \in S \cap H_{0}^{1} \cap H^{2}$ with $\left\langle z_{0}, e_{1, V}\right\rangle \neq 0$ and $0<$ $\mathcal{V}\left(z_{0}\right)<1$ there is a sequence $k_{n} \geq 1$ verifying

$$
\mathcal{U}_{k_{n}}\left(z_{0}\right) \rightarrow c e_{1, V} \text { in } H^{2}
$$

where $c \in \mathbb{C},|c|=1$.

See Subsection 3.3 for the proof of this theorem. The following lemma shows that the hypothesis on the initial condition $z_{0}$ is not restrictive.

Lemma 3.4. For any $z_{0} \in S$ there is a control $u \in C^{\infty}([0, \infty), \mathbb{R})$ and a time $k \geq 1$ such that $\left\langle\mathcal{U}_{k}\left(z_{0}, u\right), e_{1, V}\right\rangle \neq 0$.

Proof. It suffices to find a control $u$ and a time $k \geq 1$ such that

$$
\left\|\mathcal{U}_{k}\left(z_{0}, u\right)-c e_{1, V}\right\|<\sqrt{2}
$$


for some $c \in \mathbb{C},|c|=1$. Take any $\hat{z}_{0} \in S \cap H_{0}^{1} \cap H^{2}$ such that $\left\langle\hat{z}_{0}, e_{1, V}\right\rangle \neq 0$ and

$$
\left\|z_{0}-\hat{z}_{0}\right\|<\frac{\sqrt{2}}{2}
$$

By Theorem 3.3, there is a control $u \in C^{\infty}([0, \infty), \mathbb{R})$ and a time $k \geq 1$ such that

$$
\left\|\mathcal{U}_{k}\left(\hat{z}_{0}, u\right)-c e_{1, V}\right\|<\frac{\sqrt{2}}{2} .
$$

Using the fact that the $L^{2}$-distance between two solutions of (1.1), (1.2) with the same control is constant, we obtain (3.5).

\subsection{Approximate controllability}

Before proving Theorem 3.3, let us give an application of the result. For any $d>0$ define the set

$$
C_{d}=\left\{u \in C^{\infty}([0, \infty), \mathbb{R}): \sup _{t \in[0, \infty)}|u(t)|<d\right\} .
$$

We say that problem (1.1), (1.2) is approximately controllable in $L^{2}$ at integer times if for any $\varepsilon, d>0$ and for any points $z_{0}, z_{1} \in S$ there is a time $k \in \mathbb{N}$ and a control $u \in C_{d}$ such that

$$
\left\|\mathcal{U}_{k}\left(z_{0}, u\right)-z_{1}\right\|<\varepsilon .
$$

Theorem 3.5. Under Condition 3.2, problem (1.1), (1.2) is approximately controllable in $L^{2}$ at integer times.

Proof. Theorem 3.3 implies that for any $z \in S \cap H_{0}^{1} \cap H^{2}$ there is $u \in C_{d}$ such that

$$
\left\|\mathcal{U}_{k}(z, u)-e_{1, V}\right\|<\frac{\varepsilon}{2}
$$

for some $k \geq 1$. As the $L^{2}$-distance between two solutions of (1.1), (1.2) with the same control is constant, by a density argument, we get that for any $z \in S$ a control $u \in C_{d}$ exists such that (3.6) holds.

Here we need the following result often referred as time reversibility property of Schrödinger equation.

Lemma 3.6. Suppose that $\mathcal{U}_{k}(\bar{z}, w)=\bar{y}$ for some $z \in L^{2}, w \in C_{d}$ and $k \geq 1$. Then $\mathcal{U}_{k}(y, u)=z$, where $u(t)=w(k-t)$.

The proof of this lemma is clear. Let us fix any $z_{0}, z_{1} \in S$ and let $u_{0}, w \in C_{d}$ be such that

$$
\begin{array}{r}
\left\|\mathcal{U}_{k_{1}}\left(\bar{z}_{1}, w\right)-e_{1, V}\right\|<\frac{\varepsilon}{2}, \\
\left\|\mathcal{U}_{k_{0}}\left(z_{0}, u_{0}\right)-e_{1, V}\right\|<\frac{\varepsilon}{2}
\end{array}
$$


for some $k_{0}, k_{1} \geq 1$. Define $y:=\overline{\mathcal{U}_{k_{1}}\left(\bar{z}_{1}, w\right)}$. Then by Lemma 3.6, we have $\mathcal{U}_{k_{1}}\left(y, u_{1}\right)=z_{1}$, where $u_{1}(t):=w\left(k_{1}-t\right)$. Again using the fact that $L^{2}$-distance between two solutions of (1.1), (1.2) with the same control is constant, we get

$$
\left\|\mathcal{U}_{k_{1}}\left(e_{1, V}, u_{1}\right)-z_{1}\right\|=\left\|e_{1, V}-y\right\|<\frac{\varepsilon}{2} .
$$

Taking $k=k_{0}+k_{1}$ and $\hat{u}(t)=u_{0}(t), t \in\left[0, k_{0}\right)$ and $\hat{u}(t)=u_{1}\left(t-k_{0}\right)$, $t \in\left[k_{0}, \infty\right)$, we obtain

$$
\left\|\mathcal{U}_{k}\left(z_{0}, \hat{u}\right)-z_{1}\right\|<\varepsilon .
$$

Finally, using the continuity of $\mathcal{U}_{k}\left(z_{0}, \cdot\right)$, we find $u \in C_{d}$ satisfying

$$
\left\|\mathcal{U}_{k}\left(z_{0}, u\right)-z_{1}\right\|<\varepsilon .
$$

Remark 3.7. We note that for $m=1, Q(x)=x$ a stronger result is obtained by K. Beauchard and M. Mirrahimi [9] in the case of the space $L^{2}$. They show an approximate stabilization result of eigenstates. The proof of this result remains literally the same for system (1.1), (1.2) under Condition 3.2. One should just pay attention to the fact that in the case of any space dimension $m$ the spectral gap property for the eigenvalues used in [9] does not hold. The argument can be replaced by Lemma 3.10 .

\subsection{Proof of Theorem 3.3}

Step 1. Let us suppose that $u\left(\mathcal{U}_{t}\left(z_{0}\right)\right)=0$ for all $t \geq 0$. Then

$$
\mathcal{U}_{t}\left(z_{0}\right)=\sum_{j=1}^{\infty} e^{-i \lambda_{j, V} t}\left\langle z_{0}, e_{j, V}\right\rangle e_{j, V} .
$$

Substituting (3.7) into (3.2), we get

$$
\begin{aligned}
0= & \sum_{j=1, k=2}^{\infty} \alpha \lambda_{k, V}\left\langle z_{0}, e_{j, V}\right\rangle\left\langle e_{k, V}, z_{0}\right\rangle\left\langle(-\Delta+V)\left(P_{1, V}\left(Q e_{j, V}\right)\right), e_{k, V}\right\rangle e^{-i\left(\lambda_{j, V}-\lambda_{k, V}\right) t} \\
& -\sum_{j=1, k=2}^{\infty} \alpha \lambda_{k, V}\left\langle e_{j, V}, z_{0}\right\rangle\left\langle z_{0}, e_{k, V}\right\rangle\left\langle e_{k, V},(-\Delta+V)\left(P_{1, V}\left(Q e_{j, V}\right)\right)\right\rangle e^{i\left(\lambda_{j, V}-\lambda_{k, V}\right) t} \\
& -\sum_{j=1}^{\infty}\left\langle z_{0}, e_{j, V}\right\rangle\left\langle e_{1, V}, z_{0}\right\rangle\left\langle Q e_{j, V}, e_{1, V}\right\rangle e^{i\left(\lambda_{1, V}-\lambda_{j, V}\right) t} \\
& +\sum_{j=1}^{\infty}\left\langle e_{j, V}, z_{0}\right\rangle\left\langle z_{0}, e_{1, V}\right\rangle\left\langle Q e_{j, V}, e_{1, V}\right\rangle e^{-i\left(\lambda_{1, V}-\lambda_{j, V}\right) t}
\end{aligned}
$$




$$
\begin{aligned}
= & \sum_{j=2, k=2}^{\infty} P\left(z_{0}, Q, j, k\right) e^{-i\left(\lambda_{j, V}-\lambda_{k, V}\right) t} \\
+ & \sum_{j=2}^{\infty}\left[\left(\alpha \lambda_{j, V}\left\langle(-\Delta+V)\left(P_{1, V}\left(Q e_{1, V}\right)\right), e_{j, V}\right\rangle+\left\langle Q e_{j, V}, e_{1, V}\right\rangle\right)\right. \\
& \left.\times\left\langle z_{0}, e_{1, V}\right\rangle\left\langle e_{j, V}, z_{0}\right\rangle e^{i\left(\lambda_{1, V}-\lambda_{j, V}\right) t}\right] \\
- & \sum_{j=2}^{\infty}\left[\left(\alpha \lambda_{j, V}\left\langle(-\Delta+V)\left(P_{1, V}\left(Q e_{1, V}\right)\right), e_{j, V}\right\rangle+\left\langle Q e_{j, V}, e_{1, V}\right\rangle\right)\right. \\
& \left.\times\left\langle e_{1, V}, z_{0}\right\rangle\left\langle z_{0}, e_{j, V}\right\rangle e^{-i\left(\lambda_{1, V}-\lambda_{j, V}\right) t}\right],
\end{aligned}
$$

where $P\left(z_{0}, Q, j, k\right)$ is a constant. In view of Condition 3.2, (ii), Lemma 3.10 below implies that the coefficients of exponential functions in (3.8) vanish. Condition 3.2, (i), implies that the set

$$
\begin{array}{r}
J:=\left\{\alpha \in \mathbb{R}: \alpha \lambda_{j, V}\left\langle(-\Delta+V)\left(P_{1, V}\left(Q e_{j, V}\right)\right), e_{1, V}\right\rangle+\left\langle Q e_{j, V}, e_{1, V}\right\rangle=0\right. \\
\text { for some } j \geq 1\}
\end{array}
$$

is finite or countable. Thus we get that $z_{0}=c e_{1, V}$ for some $c \in \mathbb{C},|c|=1$ which is a contradiction to $\mathcal{V}\left(z_{0}\right)>0$. Thus there is a time $t_{0}>0$ such that $u\left(\mathcal{U}_{t_{0}}\left(z_{0}\right)\right) \neq 0$ and

$$
\mathcal{V}\left(\mathcal{U}_{k}\left(z_{0}\right)\right)-\mathcal{V}\left(z_{0}\right)=-\frac{2}{\delta} \int_{0}^{k} u^{2}\left(\mathcal{U}_{s}\left(z_{0}\right)\right) \mathrm{d} s<0
$$

for any $k \geq t_{0}$.

Step 2. Let $\mathcal{K}$ be the $H^{2}$-weak $\omega$-limit set of the trajectory for (3.4), (1.2) issued from $z_{0}$, i.e.

$$
\mathcal{K}=\left\{z \in H_{0}^{1} \cap H^{2}: \mathcal{U}_{k_{n}}\left(z_{0}\right) \rightarrow z \text { in } H^{2} \text { for some } k_{n} \rightarrow \infty\right\} .
$$

Let

$$
m:=\inf _{z \in \mathcal{K}} \mathcal{V}(z) .
$$

This infimum is attained, i.e. there is $e \in \mathcal{K}$ such that

$$
\mathcal{V}(e)=\inf _{z \in \mathcal{K}} \mathcal{V}(z)
$$

Indeed, take any minimizing sequence $z_{n} \in \mathcal{K}$, so that $\mathcal{V}\left(z_{n}\right) \rightarrow m$. By (3.1), $z_{n}$ is bounded in $H^{2}$. Thus, without loss of generality, we can assume that $z_{n} \rightarrow e$ in $H^{2}$. This implies that $\mathcal{V}(e) \leq \liminf _{n \rightarrow \infty} \mathcal{V}\left(z_{n}\right)=m$. Let us show that $e \in \mathcal{K}$. We can choose a sequence $k_{n} \geq 1$ such that

$$
\left\|\mathcal{U}_{k_{n}}\left(z_{0}\right)-z_{n}\right\| \leq \frac{1}{n}
$$


As $\mathcal{U}_{k_{n}}\left(z_{0}\right)$ is bounded in $H^{2}$, without loss of generality, we can suppose that $\mathcal{U}_{k_{n}}\left(z_{0}\right) \rightarrow \tilde{e}, \tilde{e} \in S \cap H_{0}^{1} \cap H^{2}$. Clearly, (3.9) implies that $e=\tilde{e}$, hence $e \in \mathcal{K}$ and $\mathcal{V}(e)=m$.

Let us show that $\mathcal{V}(e)=0$. Suppose that $\mathcal{V}(e)>0$. As $\mathcal{V}(e) \leq \mathcal{V}\left(z_{0}\right)<1$, we have $\left\langle e, e_{1, V}\right\rangle \neq 0$. Then, by Step 1 , there is a time $k \geq 1$ such that $\mathcal{V}\left(\mathcal{U}_{k}(e)\right)<$ $\mathcal{V}(e)$. Proposition 3.1 implies that $\mathcal{U}_{k}(e) \in \mathcal{K}$. This contradicts the definition of $e$. Hence $\mathcal{V}(e)=0$. Thus $e=c e_{1, V},|c|=1$ and $c e_{1, V} \in \mathcal{K}$.

Remark 3.8. We note that if there is a sequence $n_{k} \geq 1$ such that $\mathcal{U}_{n_{k}}\left(z_{0}\right)$ converges in $H^{2}$ and $z_{0}$ satisfies the hypotheses of Theorem 3.3. then the proof of the stabilization result obtained in 8 for finite-dimensional approximations of Schrödinger equation works giving

$$
\mathcal{U}_{n_{k}}\left(z_{0}, u\right) \rightarrow e_{1, V} \text { in } H^{2} .
$$

However, the existence of such a sequence is an open question.

Remark 3.9. Modifying slightly Condition 3.2. Theorem 3.3 can be restated for the eigenfunction $e_{i, V}, i \geq 1$. Indeed, one should replace $\lambda_{1, V}$ and $e_{1, V}$ by $\lambda_{i, V}$ and $e_{i, V}$ in Condition 3.2 and use the Lyapunov function

$$
\mathcal{V}_{i}(z):=\alpha\left\|(-\Delta+V) P_{i, V} z\right\|^{2}+1-\left|\left\langle z, e_{i, V}\right\rangle\right|^{2}, \quad z \in S \cap H_{0}^{1} \cap H^{2},
$$

where $P_{i, V}$ is the orthogonal projection in $L^{2}$ onto the closure of the vector span of $\left\{e_{k, V}\right\}_{k \neq i}$.

Lemma 3.10. Suppose that $r_{j} \in \mathbb{R}$ and $r_{k} \neq r_{j}$ for $k \neq j$. If

$$
\sum_{j=1}^{\infty} c_{j} e^{i r_{j} t}=0
$$

for any $t \geq 0$ and for some sequence $c_{j} \in \mathbb{C}$ such that $\sum_{j=1}^{\infty}\left|c_{j}\right|<\infty$, then $c_{j}=0$ for all $j \geq 1$.

Proof. Multiplying (3.10) by $e^{-i r_{n} t}$ and integrating on the interval $[0, T]$, we get

$$
c_{n}=-\frac{1}{T} \sum_{j=1, j \neq n}^{\infty} c_{j} \int_{0}^{T} e^{i\left(r_{j}-r_{n}\right) t} \mathrm{~d} t=-\frac{1}{T} \sum_{j=1, j \neq n}^{\infty} c_{j} \frac{e^{i\left(r_{j}-r_{n}\right) T}-1}{i\left(r_{j}-r_{n}\right)} \rightarrow 0
$$

as $T \rightarrow \infty$, by the Lebesgue theorem on dominated convergence.

\subsection{Genericity of Condition 3.2}

Let us recall some definitions. Let $X$ be a complete metric space and $A \subset X$. Then $A$ is said to be a $G_{\delta}$ set if it is a countable intersection of dense open sets. It follows from the Baire theorem that any $G_{\delta}$ subset is dense. A set $B \subset X$ is called residual if it contains a $G_{\delta}$ subset. 
Example 3.11. Let us endow the space $C^{\infty}(\bar{D}, \mathbb{R})$ with its usual topology given by the countable family of norms:

$$
p_{n}(Q):=\sum_{|\alpha| \leq n} \sup _{x \in D}\left|\partial^{\alpha} Q(x)\right| .
$$

The set $\mathcal{P}$ of all functions $Q \in C^{\infty}(\bar{D}, \mathbb{R})$ such that $\left\langle Q e_{1, V}, e_{j, V}\right\rangle \neq 0$ for all $j \geq 1$ is $G_{\delta}$. Indeed, let us fix an integer $j \geq 1$ and let $\mathcal{P}_{j}$ be the set of functions $Q \in C^{\infty}(\bar{D}, \mathbb{R})$ verifying $\left\langle Q e_{1, V}, e_{j, V}\right\rangle \neq 0$. The unique continuation theorem for the operator $-\Delta+V$ (see [18]) implies that there is a ball $B \subset D$ such that $e_{1, V}(x) e_{j, V}(x) \neq 0$ for all $x \in B$. Let $Q \in C^{\infty}(\bar{D}, \mathbb{R})$ be such that $Q \neq 0$, $\operatorname{supp} Q \subset B$ and $Q \geq 0$. Then $Q \in \mathcal{P}_{j}$, hence $\mathcal{P}_{j}$ is non-empty. Clearly, $\mathcal{P}_{j}$ is open. Take any $Q_{1} \in C^{\infty}(\bar{D}, \mathbb{R})$ such that $\left\langle Q_{1} e_{1, V}, e_{j, V}\right\rangle=0$ and $Q_{2} \in \mathcal{P}_{j}$. Then $\left\langle\left(Q_{1}+\tau Q_{2}\right) e_{1, V}, e_{j, V}\right\rangle \neq 0$ for all $\tau \neq 0$. Thus $\mathcal{P}_{j}$ is dense in $C^{\infty}(\bar{D}, \mathbb{R})$ and $\mathcal{P}=\cap_{j=1}^{\infty} \mathcal{P}_{j}$ is a $G_{\delta}$ set.

The following lemma shows that property (ii) of Condition 3.2 is generic in $1 \mathrm{D}$ case.

Lemma 3.12. Let $I \subset \mathbb{R}$ be an interval and let $\mathcal{Q}$ be the set of all functions $V \in C^{\infty}(I, \mathbb{R})$, verifying

$$
\lambda_{i, V}-\lambda_{j, V} \neq \lambda_{p, V}-\lambda_{q, V}
$$

for all $i, j, p, q \geq 1$ such that $\{i, j\} \neq\{p, q\}$ and $i \neq j$. Then $\mathcal{Q}$ is a $G_{\delta}$ set.

Proof. It is well known that the spectrum $\left\{\lambda_{j, V}\right\}$ of $-\frac{\mathrm{d}^{2}}{\mathrm{~d} x^{2}}+V$ is non-degenerate for any $V \in C^{\infty}(\bar{D}, \mathbb{R})$, and $e_{j, V}$ and $\lambda_{j, V}$ are real-analytic in $V$ (e.g., see [24]). Let us introduce the set $\mathcal{Q}_{n}, n \geq 1$ of all functions $V \in C^{\infty}(\bar{D}, \mathbb{R})$ such that (3.11) is satisfied for any $1 \leq i, j, p, q \leq n$. Clearly,

$$
\mathcal{Q}=\bigcap_{n=1}^{\infty} \mathcal{Q}_{n}
$$

It suffices to prove that $\mathcal{Q}_{n}$ is open and dense in $C^{\infty}(\bar{D}, \mathbb{R})$. The fact that $\mathcal{Q}_{n}$ is open follows directly from the continuity of $\lambda_{j, V}$ in $V$. Let us prove that $\mathcal{Q}_{n}$ is dense in $C^{\infty}(\bar{D}, \mathbb{R})$.

Take any $1 \leq i, j, p, q \leq n$ such that $\{i, j\} \neq\{p, q\}$ and $i \neq j$, and let $\mathcal{Q}_{i, j, p, q}$ be the set of functions $V \in C^{\infty}(\bar{D}, \mathbb{R})$ such that (3.11) is satisfied. Suppose we have proved that for any $V \in C^{\infty}(\bar{D}, \mathbb{R})$ there is $\sigma \in C^{\infty}(\bar{D}, \mathbb{R})$ such that

$$
\lambda_{i, V+\tau \sigma}-\lambda_{j, V+\tau \sigma} \neq \lambda_{p, V+\tau \sigma}-\lambda_{q, V+\tau \sigma},
$$

for any small $\tau>0$. This implies that $\mathcal{Q}_{i, j, p, q}$ is dense. On the other hand, $\mathcal{Q}_{i, j, p, q}$ is open. Hence $\mathcal{Q}_{n}$ is dense, as

$$
\mathcal{Q}_{n}=\bigcap_{1 \leq i, j, p, q \leq n} \mathcal{Q}_{i, j, p, q}
$$


To prove (3.12), following [2], let us write

$$
\begin{aligned}
\lambda_{j, V+\tau \sigma} & =\lambda_{j, V}+\alpha_{j} \tau+\beta_{j}(\tau) \tau^{2}, \\
e_{j, V+\tau \sigma} & =e_{j, V}+v_{j} \tau+w_{j}(\tau) \tau^{2} .
\end{aligned}
$$

Differentiating the identity

$$
\left(-\frac{\mathrm{d}^{2}}{\mathrm{~d} x^{2}}+V+\tau \sigma-\lambda_{j, V+\tau \sigma}\right) e_{j, V+\tau \sigma}=0
$$

with respect to $\tau$ at $\tau=0$ and using (3.13) and (3.14), we get

$$
\left(-\frac{\mathrm{d}^{2}}{\mathrm{~d} x^{2}}+V-\lambda_{j, V}\right) v_{j}+\left(\sigma-\alpha_{j}\right) e_{j, V}=0
$$

Taking the scalar product of this identity with $e_{j, V}$, we obtain

$$
\left\langle\sigma,\left(e_{j, V}\right)^{2}\right\rangle=\alpha_{j} .
$$

Suppose that

$$
\lambda_{i, V+\tau \sigma}-\lambda_{j, V+\tau \sigma}=\lambda_{p, V+\tau \sigma}-\lambda_{q, V+\tau \sigma}
$$

for any $\sigma \in C^{\infty}(\bar{D}, \mathbb{R})$ and for some sequence $\tau_{n} \rightarrow 0$. Clearly, this implies that

$$
\alpha_{i}-\alpha_{j}=\alpha_{p}-\alpha_{q}
$$

In view of (3.15), this gives

$$
\left(e_{i, V}\right)^{2}-\left(e_{j, V}\right)^{2}=\left(e_{p, V}\right)^{2}-\left(e_{q, V}\right)^{2} .
$$

On the other hand, by Theorem 9 in 24 (see page 46), the system $\left\{\left(e_{n, V}\right)^{2}\right\}$ is independent for any $V \in L^{2}$. This contradiction proves (3.12) and completes the proof of the lemma.

We now turn to the multidimensional case. Let us assume that $D=[0,1]^{n}$ and introduce the space

$$
\begin{aligned}
\mathcal{G}:=\left\{V \in C^{\infty}(D, \mathbb{R}): V\left(x_{1}, \ldots, x_{n}\right)=V_{1}\left(x_{1}\right)+\ldots+V_{n}\left(x_{n}\right)\right. \\
\left.\quad \text { for some } V_{k} \in C^{\infty}([0,1], \mathbb{R}), k=1, \ldots, n\right\} .
\end{aligned}
$$

Endow $\mathcal{G}$ with the metric of $C^{\infty}(D, \mathbb{R})$. It is not difficult to verify that $\mathcal{G}$ is a closed subspace in $C^{\infty}(D, \mathbb{R})$.

Lemma 3.13. The set of all functions $V \in \mathcal{G}$, verifying

$$
\lambda_{i, V}-\lambda_{j, V} \neq \lambda_{p, V}-\lambda_{q, V}
$$

for all $i, j, p, q \geq 1$ such that $\{i, j\} \neq\{p, q\}$ and $i \neq j$, is a $G_{\delta}$ set. 
Proof. Notice that any eigenfunction of $-\Delta+V, V \in \mathcal{G}$ has the form

$$
e_{l, V}\left(x_{1}, \ldots, x_{n}\right)=e_{l_{1}, V_{1}}\left(x_{1}\right) \cdot \ldots \cdot e_{l_{n}, V_{n}}\left(x_{n}\right),
$$

where $e_{l_{k}, V_{k}}\left(x_{k}\right)$ is an eigenfunction of the operator $-\frac{\mathrm{d}^{2}}{\mathrm{~d} x_{k}^{2}}+V_{k}$. Indeed, any function of form (3.18) is an eigenfunction, and the set of all functions of this form is a basis in $L^{2}(D)$.

Let $i, j, p, q \geq 1$ be such that $\{i, j\} \neq\{p, q\}$ and $i \neq j$, and let $e_{i_{n}, V_{n}}\left(x_{n}\right)$, $e_{j_{n}, V_{n}}\left(x_{n}\right), e_{p_{n}, V_{n}}\left(x_{n}\right)$ and $e_{q_{n}, V_{n}}\left(x_{n}\right)$ be the eigenfunctions in (3.18). Without loss of generality, we can suppose that the functions $\left(e_{i_{n}, V_{n}}\left(x_{n}\right)\right)^{2},\left(e_{j_{n}, V_{n}}\left(x_{n}\right)\right)^{2}$, $\left(e_{p_{n}, V_{n}}\left(x_{n}\right)\right)^{2}$ and $\left(e_{q_{n}, V_{n}}\left(x_{n}\right)\right)^{2}$ are linearly independent (see Theorem 9 in [24). Any eigenfunction $e_{l_{k}, V_{k}}$ has a finite number of zeros in interval $[0,1]$. Hence, choosing appropriately the point $x^{*} \in[0,1]^{n-1}$, we see that the functions $\left(e_{i, V}\left(x^{*}, x_{n}\right)\right)^{2},\left(e_{j, V}\left(x^{*}, x_{n}\right)\right)^{2},\left(e_{p, V}\left(x^{*}, x_{n}\right)\right)^{2}$ and $\left(e_{q, V}\left(x^{*}, x_{n}\right)\right)^{2}, x_{n} \in[0,1]$ are linearly independent. This implies that relation (3.16) does not hold. Thus the proof of Lemma 3.12 works implying the genericity.

\section{Applications}

\subsection{Nonlinear Schrödinger equation}

Let us consider the nonlinear Schrödinger equation

$$
\begin{aligned}
i \dot{z} & =-\Delta z+V(x) z+u(t) Q(x)|z|^{2} z, \quad x \in D, \\
\left.z\right|_{\partial D} & =0, \\
z(0, x) & =z_{0}(x),
\end{aligned}
$$

where $D \subset \mathbb{R}^{3}$ is a bounded domain with smooth boundary. Problem (4.1)-(4.3) is locally well-posed.

Lemma 4.1. For any $z_{0} \in H_{0}^{1} \cap H^{2}$ and for any $u \in L_{l o c}^{1}([0, \infty), \mathbb{R})$ there is a time $T>0$ such that problem (4.1)-(4.3) has a unique solution $z \in$ $C\left([0, T], H^{2}\right)$. Furthermore, the resolving operator $\mathcal{U}_{t}(\cdot, u): H_{0}^{1} \cap H^{2} \rightarrow H_{0}^{1} \cap H^{2}$ taking $z_{0}$ to $z(t)$ satisfies the relation

$$
\left\|\mathcal{U}_{t}\left(z_{0}, u\right)\right\|=\left\|z_{0}\right\|, \quad t \in[0, T] .
$$

See 12 for the proof. Define $z(t)=\mathcal{U}_{t}\left(z_{0}, u\right)$ and let us calculate the derivative

$$
\begin{aligned}
\frac{\mathrm{d}}{\mathrm{d} t} \mathcal{V}(z(t))= & 2 \alpha \operatorname{Re}\left(\left\langle(-\Delta+V) P_{i, V} \dot{z}, P_{i, V}(-\Delta+V) z\right\rangle\right)-2 \operatorname{Re}\left(\left\langle\dot{z}, e_{i, V}\right\rangle\left\langle e_{i, V}, z\right\rangle\right) \\
= & 2 \alpha \operatorname{Re}\left(\left\langle(-\Delta+V) P_{i, V}\left(i \Delta z-i V z-i u Q|z|^{2} z\right),(-\Delta+V) P_{i, V} z\right\rangle\right) \\
& -2 \operatorname{Re}\left(\left\langle i \Delta z-i V z-i u Q|z|^{2} z, e_{i, V}\right\rangle\left\langle e_{i, V}, z\right\rangle\right) \\
= & 2 u \operatorname{Im}\left(\alpha\left\langle(-\Delta+V) P_{i, V}\left(Q|z|^{2} z\right),(-\Delta+V) P_{i, V} z\right\rangle\right. \\
& \left.-\left\langle Q|z|^{2} z, e_{i, V}\right\rangle\left\langle e_{i, V}, z\right\rangle\right) .
\end{aligned}
$$


Take

$u(z):=-\operatorname{Im}\left(\left\langle\alpha(-\Delta+V) P_{i, V}\left(Q|z|^{2} z\right),(-\Delta+V) P_{i, V} z\right\rangle-\left\langle Q|z|^{2} z, e_{i, V}\right\rangle\left\langle e_{i, V}, z\right\rangle\right)$.

Problem (4.1)-(4.3) with feedback (4.4) is globally well-posed in $H^{2}$ (cf. Theorem 3.1). Let $\mathcal{U}_{t}(\cdot): H_{0}^{1} \cap H^{2} \rightarrow H_{0}^{1} \cap H^{2}$ be the resolving operator. To formulate the main result, we introduce the following hypothesis.

Condition 4.2. The functions $V, Q \in C^{\infty}(\bar{D}, \mathbb{R})$ are such that:

(i) $\left\langle Q e_{i, V} e_{j, V}, e_{p, V} e_{q, V}\right\rangle \neq 0$ for all $i, j, p, q \geq 1$,

(ii) $\lambda_{i, V}-\lambda_{j, V}+\lambda_{p, V}-\lambda_{q, V} \neq \lambda_{i^{\prime}, V}-\lambda_{j^{\prime}, V}+\lambda_{p^{\prime}, V}-\lambda_{q^{\prime}, V}$ for all integers $i, j, p, q, i^{\prime}, j^{\prime}, p^{\prime}, q^{\prime}$ such that $\{i, j, p, q\} \neq\left\{i^{\prime}, j^{\prime}, p^{\prime}, q^{\prime}\right\}$ and $\{i, p\} \neq\{j, q\}$.

The theorem below is the version of Theorem 3.3 for system (4.1)-(4.3).

Theorem 4.3. Under Condition 4.2, there is a finite or countable set $J \subset \mathbb{R}_{+}^{*}$ such that for any $\alpha \notin J, l \geq 1$ and $z_{0} \in S \cap H_{0}^{1} \cap H^{2}$ with $\left\langle z_{0}, e_{l, V}\right\rangle \neq 0$ and $0<\mathcal{V}_{l}\left(z_{0}\right)<1$ there is a sequence $k_{n} \geq 1$ verifying

$$
\mathcal{U}_{k_{n}}\left(z_{0}\right) \rightarrow c e_{l, V} \text { in } H^{2},
$$

where $c \in \mathbb{C},|c|=1$.

The proof of this theorem is very close to that of Theorem 3.3. One should notice that, under Condition 4.2 , there is a time $t_{0}>0$ such that $u\left(\mathcal{U}_{t_{0}}\left(z_{0}, 0\right)\right) \neq 0$ and then conclude as in Step 2 in the proof of Theorem 3.3

Remark 4.4. Notice that, as equation (4.1) is nonlinear, the distance between two solutions with the same control is not constant. Hence the proof of approximate controllability given in Theorem 3.5 does not work here.

Lemma 4.5. For any $l \geq 1, d>0$ and $z_{0} \in S$ there is a control $u \in C_{d}$ and $a$ time $k \geq 1$ such that $\left\langle\mathcal{U}_{k}\left(z_{0}, u\right), e_{l, V}\right\rangle \neq 0$.

Proof. Suppose that $\left\langle z_{0}, e_{l, V}\right\rangle=0$. Let us show that there is a control $u \in C_{d}$ such that $\left\langle\mathcal{U}_{k}\left(z_{0}, u\right), e_{l, V}\right\rangle \neq 0$ for some $k \geq 0$. As (4.1) is nonlinear, the proof given in Lemma 3.4 does not work.

If $z_{0} \notin\left\{c e_{j, V}: c \in \mathbb{C},|c|=1, j \geq 1\right\}$, then, by Theorem 4.3, there is an integer $p \geq 1$, sequence $k_{n} \geq 1$ and constant $c \in \mathbb{C},|c|=1$ such that $\mathcal{U}_{k_{n}}\left(z_{0}\right) \rightarrow c e_{p, V}$ in $H^{2}$. Hence, without loss of generality, we can suppose that $z_{0}=e_{p, V}$ for some $p \neq l$. Let us introduce the following two-dimensional subspace of $L^{2}([0,1], \mathbb{R})$ :

$$
E=\left\{a \sin \left(\lambda_{p, V}-\lambda_{l, V}\right) t+b \cos \left(\lambda_{p, V}-\lambda_{l, V}\right) t: a, b \in \mathbb{R}\right\} .
$$

For any $u \in E$, define the mapping $\Phi(u)=\left\langle\mathcal{U}_{1}\left(e_{p, V}, u\right), e_{l, V}\right\rangle$, whenever the solution $\mathcal{U}_{t}\left(e_{p, V}, u\right)$ exists up to time $t=1$. Notice that $\Phi(0)=\left\langle e^{-i \lambda_{p, V}} e_{p, V}, e_{l, V}\right\rangle=$ 0 , hence $\Phi$ is well defined in a neighborhood of $0 \in E$. We are going to show 
that the conditions of inverse mapping theorem are satisfied in a neighborhood of the point $0 \in E$. Clearly, $\Phi$ is continuously differentiable. Let us show that mapping $D \Phi(0): E \rightarrow \mathbb{C}$ is an isomorphism. Consider the linearization of (4.1), (4.2), $z_{0}=e_{p, V}$ around $\left(e^{-i \lambda_{p, V} t} e_{p, V}, 0\right)$ :

$$
\begin{aligned}
i \dot{y} & =-\Delta y+V(x) y+u(t) Q(x) e_{p, V}^{3} e^{-i \lambda_{p}, V t}, \quad x \in D . \\
\left.y\right|_{\partial D} & =0, \\
y(0) & =0 .
\end{aligned}
$$

One can verify that $D \Phi(0)(u)=\left\langle y(1), e_{l, V}\right\rangle$. System (4.5)-(4.7) is equivalent to

$$
y=-i \int_{0}^{t} e^{-i \lambda_{p, V} s} u(s) S(t-s)\left(Q e_{p, V}^{3}\right) \mathrm{d} s
$$

where $S(t)$ is the unitary group associated with $i \Delta-i V$. Taking the scalar product of (4.8) with $e_{l, V}$, we obtain for $t=1$

$$
\left\langle y, e_{l, V}\right\rangle=-i e^{-i \lambda_{l, V}}\left\langle Q e_{p, V}^{3}, e_{l, V}\right\rangle \int_{0}^{1} e^{-i\left(\lambda_{p, V}-\lambda_{l, V}\right) s} u(s) \mathrm{d} s .
$$

Condition 4.2 implies that $\lambda_{p, V}-\lambda_{l, V} \neq 0$, hence $D \Phi(0): E \rightarrow \mathbb{C}$ is an isomorphism. Applying the inverse mapping theorem, we conclude that $\Phi$ is $C^{1}$ diffeomorphism in a neighborhood of $0 \in E$. Thus there is a control $u \in C_{d}$ such that $\left\langle\mathcal{U}_{1}\left(e_{p, V}, u\right), e_{l, V}\right\rangle \neq 0$.

\subsection{Randomly forced Schrödinger equation}

\subsubsection{Growth of Sobolev norms}

Let us consider the problem

$$
\begin{aligned}
i \dot{z} & =-\Delta z+V(x) z+\beta(t) Q(x) z, \quad x \in D, \\
\left.z\right|_{\partial D} & =0, \\
z(0) & =z_{0},
\end{aligned}
$$

where $V, Q \in C^{\infty}(\bar{D}, \mathbb{R})$ are given functions. We assume that $\beta(t)$ is a random process of the form (2.2), where the random variables $\eta_{k}$ verify the following condition.

Condition 4.6. The random variables $\eta_{k}$ have the form

$$
\eta_{k}(t)=\sum_{j=1}^{\infty} b_{j} \xi_{j k} g_{j}(t), \quad t \in[0,1]
$$

where $\left\{g_{j}\right\}$ is an orthonormal basis in $L^{2}([0,1], \mathbb{R}), b_{j}>0$ are constants with

$$
\sum_{j=1}^{\infty} b_{j}^{2}<\infty
$$


and $\xi_{j k}$ are independent real-valued random variables such that $\mathbb{E} \xi_{j k}^{2}=1$. Moreover, the distribution of $\xi_{j k}$ possesses a continuous density $\rho_{j}$ with respect to the Lebesgue measure and $\rho_{j}(r)>0$ for all $r \in \mathbb{R}$.

Notice that this condition in particular implies that

$$
\mathbb{P}\left\{\|u-\beta\|_{L^{2}([0, l])}<\varepsilon\right\}>0
$$

for any $u \in L^{2}([0, l])$ and $\varepsilon>0$. Moreover, using the continuity of the mapping $\mathcal{U}_{l}\left(z_{0}, \cdot\right): L^{2}([0, l]) \rightarrow L^{2}(D)$, for any $\delta>0$ we can find a constant $\varepsilon>0$ such that

$$
\mathbb{P}\left\{\left\|\mathcal{U}_{l}\left(z_{0}, \beta\right)-\mathcal{U}_{l}\left(z_{0}, u\right)\right\|<\delta\right\} \geq \mathbb{P}\left\{\|u-\beta\|_{L^{2}([0, l])}<\varepsilon\right\}>0 .
$$

Hence, any point $\mathcal{U}_{l}\left(z_{0}, u\right), u \in L^{2}([0, l])$ is in the support of the measure $\mathcal{D}\left(\mathcal{U}_{l}\left(z_{0}, \beta\right)\right)$.

The following theorem is the main result of this section.

Theorem 4.7. Suppose that Conditions 3.2 and 4.6 are satisfied. Then for any $s>0$ and $z \in H^{s} \backslash\{0\}$ we have

$$
\mathbb{P}\left\{\limsup _{k \rightarrow \infty}\left\|\mathcal{U}_{k}(z, \beta)\right\|_{s}=\infty\right\}=1 .
$$

\subsubsection{Proof of Theorem 4.7}

By Theorem 3.5, system (1.1), (1.2) is approximately controllable at integer times. Since the equation is linear in $z$, it suffices to prove (4.12) for any $z \in S \cap H^{s}$. Without loss of generality, we can assume that $s \in(0,2]$.

Step 1. Let us fix a constant $r>0$ and introduce the stopping time

$$
\tau_{r}(z)=\min \left\{k \geq 0: \mathcal{U}_{k}(z, \beta) \in B_{H^{-s}}(0, r)\right\}, \quad z \in B_{L^{2}}(0,1) .
$$

Then we have

$$
\mathbb{P}\left\{\tau_{r}(z)<\infty\right\}=1
$$

Indeed, choose an arbitrary point $z^{\prime} \in S \cap B_{H^{-s}}(0, r)$. By the property of approximate controllability in $L^{2}$, there is a control $u \in C_{d}$ such that $\mathcal{U}_{l}(z, u)$ is sufficiently close to $z^{\prime}$ in $L^{2}$, hence $\mathcal{U}_{l}(z, u) \in B_{H^{-s}}(0, r)$. As $\mathcal{U}_{l}(z, u)$ is in the support of measure $\mathcal{D}\left(\mathcal{U}_{l}(z, \beta)\right)$, we have

$$
\mathbb{P}\left\{\mathcal{U}_{l}(z, \beta) \in B_{H^{-s}}(0, r)\right\}>0 .
$$

Using the continuity of the resolving operator in negative Sobolev norms, we see that there is an $H^{-s}$-neighborhood $\mathcal{O}=\mathcal{O}(z)$ of $z$ such that

$$
\sup _{y \in \mathcal{O}} \mathbb{P}\left\{\tau_{r}(y)>l\right\}<1
$$

From the compactness of $B_{L^{2}}(0,1)$ in $H^{-s}$ it follows that there is a time $k \geq 1$ such that

$$
a:=\sup _{y \in B_{L^{2}}(0,1)} \mathbb{P}\left\{\tau_{r}(y)>k\right\}<1
$$


Using the Markov property and (4.14), we obtain

$$
\begin{aligned}
\mathbb{P}\left\{\tau_{r}(y)>n k\right\} & =\mathbb{E}\left(\left.I_{\left\{\tau_{r}(y)>(n-1) k\right\}} \mathbb{P}\left\{\tau_{r}(x)>k\right\}\right|_{x=\mathcal{U}_{(n-1) k}(y, \beta)}\right) \\
& \leq a \mathbb{P}\left\{\tau_{r}(y)>(n-1) k\right\} .
\end{aligned}
$$

Hence

$$
\mathbb{P}\left\{\tau_{r}(y)>n k\right\} \leq a^{n} .
$$

Using the Borel-Cantelli lemma, we arrive at (4.13).

Step 2. Take any $z \in S \cap H^{s}$. Choosing $r=\frac{1}{n}$ and using (4.13), we get

$$
\mathbb{P}\left\{\liminf _{k \rightarrow \infty}\left\|\mathcal{U}_{k}(z, \beta)\right\|_{-s}=0\right\}=1 .
$$

Define the event

$$
\mathcal{A}:=\left\{\omega \in \Omega: \limsup _{k \rightarrow \infty}\left\|\mathcal{U}_{k}(z, \beta)\right\|_{s}<\infty\right\}
$$

Suppose that

$$
\mathbb{P}\{\mathcal{A}\}>0 .
$$

By (4.16), for almost any $\omega \in \mathcal{A}$ there is a sequence $n_{k} \rightarrow \infty$ such that

$$
\lim _{n \rightarrow \infty}\left\|\mathcal{U}_{n_{k}}(z, \beta)\right\|_{-s}=0 .
$$

On the other hand, for any $\omega \in \mathcal{A}$, there is a subsequence of $n_{k}$ (which is also denoted by $n_{k}$ ) and an element $w \in S$ such that

$$
\left\|\mathcal{U}_{n_{k}}(z, \beta)-w\right\| \rightarrow 0 .
$$

This contradicts (4.17). Thus $\mathbb{P}\{\mathcal{A}\}=0$.

Remark 4.8. In view of Theorem 4.3, under Condition 4.2, Theorem 4.7 holds also in the case of nonlinear equation (4.1). The proof is literally the same. One should just pay attention to the fact that, as in this case finite time blow-up is possible, the restriction of the solution at integer times forms a Markov chain with values in $H^{s} \cup\{\infty\}$ (e.g., see [26]).

\section{References}

[1] A. Agrachev and T. Chambrion. An estimation of the controllability time for single-input systems on compact Lie groups. J. ESAIM Control Optim. Calc. Var., 12(3):409-441, 2006.

[2] J. H. Albert. Genericity of simple eigenvalues for elliptic PDE's. Proc. Amer. Math. Soc., 48:413-418, 1975.

[3] F. Albertini and D. D'Alessandro. Notions of controllability for bilinear multilevel quantum systems. IEEE Transactions on Automatic Control, 48(8):1399-1403, 2003. 
[4] C. Altafini. Controllability of quantum mechanical systems by root space decomposition of su(n). J. of Math. Phys., 43(5):2051-2062, 2002.

[5] J. M. Ball, J. E. Marsden, and M. Slemrod. Controllability for distributed bilinear systems. SIAM J. Control Optim., 20:575-597, 1982.

[6] L. Baudouin and J.-P. Puel. Uniqueness and stability in an inverse problem for the Schrödinger equation. Inverse Problems, 18:1537-1554, 2001.

[7] K. Beauchard. Local Controllability of a 1-D Schrödinger equation. J. Math. Pures et Appl., 84(7):851-956, 2005.

[8] K. Beauchard, J.-M. Coron, M. Mirrahimi, and P. Rouchon. Implicit Lyapunov control of finite dimensional Schrödinger equations. Systems and Control Letters, 56:388-395, 2007.

[9] K. Beauchard and M. Mirrahimi. Approximate stabilization of a quantum particle in a 1D infinite square potential well. Submitted, 2007.

[10] J. Bourgain. On growth of Sobolev norms in linear Schrödinger equations with smooth time dependent potential. J. Anal. Math., 77:315-348, 1999.

[11] N. Burq. Contrôle de l'équation des plaques en présence d'obstacles strictement convexes. Mémoire de la S.M.F., 55, 1993.

[12] T. Cazenave. Semilinear Schrödinger equations. Courant Lecture Notes in Mathematics, AMS, 10, 2003.

[13] T. Chambrion, P. Mason, M. Sigalotti, and U. Boscain. Controllability of the discrete-spectrum Schrödinger equation driven by an external field. Preprint, 2008.

[14] J.-M. Coron. Control and nonlinearity. Mathematical Surveys and Monographs, American Mathematical Society, Providence, RI, 136, 2007.

[15] B. Dehman, P. Gérard, and G. Lebeau. Stabilization and control for the nonlinear Schrödinger equation on a compact surface. Math. Z., 254(4):729749, 2006.

[16] L. H. Eliasson and S. B. Kuksin. On reducibility of linear Schrödinger equations with quasiperiodic potentials. Preprint, 2007.

[17] M. B. Erdogan, R. Killip, and W. Schlag. Energy growth in Schrödinger's equation with Markovian forcing. Comm. Math. Phys., 240:1-29, 2003.

[18] D. Jerison and C. E. Kenig. Unique continuation and absence of positive eigenvalues for Schrödinger operators (with an appendix by E. M. Stein). Ann. of Math., 121(3):463-494, 1985.

[19] G. Lebeau. Contrôle de l'équation de Schrödinger. J. Math. Pures Appl., 71:267-291, 1992. 
[20] E. Machtyngier and E. Zuazua. Stabilization of the Schrödinger equation. Portugaliae Matematica, 51(2):243-256, 1994.

[21] M. Mirrahimi. Lyapunov control of a particle in a finite quantum potential well. IEEE Conf. on Decision and Control, San Diego, 2006.

[22] V. Nersesyan. Exponential mixing for finite-dimensional approximations of the Schrödinger equation with multiplicative noise. Submitted, 2007.

[23] B. Øksendal. Stochastic Differential Equations. Springer-Verlag, 2003.

[24] J. Pöschel and E. Trubowitz. Inverse Spectral Theory. Academic Press, New York, 1987.

[25] V. Ramakrishna, M. Salapaka, M. Dahleh, H. Rabitz, and A. Pierce. Controllability of molecular systems. Phys. Rev. A, 51(2):960-966, 1995.

[26] D. Revuz. Markov Chains. North-Holland, Amsterdam, 1984.

[27] G. Turinici and H. Rabitz. Quantum wavefunction controllability. Chem. Phys., 267:1-9, 2001.

[28] E. Zuazua. Remarks on the controllability of the Schrödinger equation. CRM Proc. Lecture Notes, 33:193-211, 2003. 